\title{
COMPARTILHAMENTO DO CONHECIMENTO EM INCUBADORAS BRASILEIRAS ASSOCIADAS À
}

ANPROTEC

SHARING OF KNOWLEDGE IN BRAZILIAN INCUBATORS ASSOCIATED TO ANPROTEC

FABIANO MAURY RAUPP

Mestre em Administração - CPGA/UFSC.

Professor do Departamento de Estudos Contábeis e Financeiros da Escola Superior de Administração e Gerência (Esag) da Universidade do Estado de Santa Catarina (Udesc). Av. Madre Benvenuta, 2037, Itacorubi - Florianópolis - SC - CEP 88035-001 E-mail: fabianoraupp@hotmail.com

ILSE MARIA BEUREN

Doutora em Contabilidade e Controladoria pela FEA/USP. Professora do Programa de Pós-Graduação em Ciências Contábeis (PPGCC) da Universidade Regional de Blumenau (Furb).

Rua Antônio da Veiga, 140, Victor Konder (Campus I - sala D-206) - Blumenau - SC - CEP 89010-500 E-mail: ilse@furb.br 


\section{RESUMO}

Este artigo objetiva descrever o processo de compartilhamento do conhecimento em incubadoras brasileiras associadas à Associação Nacional de Entidades Promotoras de Empreendimentos de Tecnologias Avançadas (Anprotec). Para tanto, primeiramente, discorreu-se sobre a gestão do conhecimento, o compartilhamento do conhecimento nas organizações e a conceituação de incubadoras de empresas. Em seguida, procedeu-se ao estudo de levantamento ou survey, analisando o compartilhamento do conhecimento em 33 incubadoras brasileiras associadas à Anprotec, conforme relação disponível em seu site. Os dados foram coletados por meio de questionários enviados por correio eletrônico, com um retorno de I8\%. Os resultados da pesquisa indicaram que há uma preocupação em gerar, difundir e, sobretudo, compartilhar informações e conhecimentos nas incubadoras estudadas. Quanto às formas de compartilhamento analisadas, verificou-se que as palestras, as reuniões, os encontros e as discussões são os eventos que apresentam maior grau de importância nas incubadoras. Por fim, observou-se que as incubadoras buscam pessoas especializadas para a realização de palestras ou seminários, bem como incentivam a participação dos empregados em encontros regionais e nacionais.

\section{PALAVRAS-CHAVE}

Compartilhamento do conhecimento; Incubadoras brasileiras; Anprotec.

\section{ABSTRACT}

The objective of this article is to describe the process of sharing knowledge in Brazilian incubators associated to the National Association of Promoting Entities of Enterprises of Advanced Technologies (Anprotec). As a first step, a panoramic view on the management of knowledge, the sharing of knowledge in organizations 
and the conceptualization of the incubators of companies is presented. After that, an analysis of the data collected is undertaken, which covers the sharing of knowledge in 33 Brazilian incubators associated to the Anprotec. The list of companies was available in the site of Anprotec and the data were collected through questionnaires sent by e-mail, which had a I $8 \%$ return. The results of the research indicate that there is a concern with generating, spreading and, above all, sharing information and knowledge on the part of the incubators scrutinized. As to the strategies of sharing, it was verified that lectures, meetings and discussions are the most important ones. Finally, it was observed that the incubators search for people specialized in lecturing or supervising seminars for those events, and stimulate the participation of their employees in regional and national conferences.

\section{KEYWORDS}

Sharing of knowledge; Brazilian incubators; Anprotec.

\section{INTRODUÇÃO}

Atualmente, o cenário mundial presencia a ocorrência de três revoluções simultâneas no mercado: econômica, administrativa e tecnológica. Essas revoluções têm causado impacto significativo no ambiente empresarial, resultando em profundas alterações tanto no contexto macrossocial das relações contratuais das organizações como no ambiente microssocial das empresas. Essa conjuntura tem incentivado o desenvolvimento de novas formas de gestão, com modelos mais flexíveis, permitindo que as empresas tenham a capacidade de mudar tanto quanto lhes é exigido.

Dentre os vários segmentos empresariais, as micro e pequenas empresas têm-se destacado no contexto organizacional, à medida que contribuem para o crescimento econômico do País e para a geração de novos empregos. Sobre a importância das micro e pequenas empresas, Fonseca e Kruglianskas (2000, p. 2) explicitam que:

as pequenas empresas passaram a representar, no final do século 20, a esmagadora maioria de unidades de negócio em todos os países do mundo, fossem industrializados, em desenvolvimento ou subdesenvolvidos. Estas passaram a responder por cerca de 50\%, quando não mais do valor da produção e a reter mais da metade do total dos postos de trabalho. 
Essas empresas, no entanto, apresentam algumas dificuldades na condução do negócio, destacando-se entre elas: problemas na obtenção de linhas de financiamento, inexistência de planejamento de longo prazo, falta de apoio no desenvolvimento do produto e geralmente há ausência de uma formação gerencial. Para solucionar parte desses problemas, surgiram organizações com o intuito de oferecer um conjunto de mecanismos que facilitam o desenvolvimento e a inserção dessas empresas no mercado, como as incubadoras de empresas.

Assim, um dos focos de interesse está na maneira como se dá o compartilhamento do conhecimento a partir do momento em que este é gerado. Nesse sentido, este artigo tem como objetivo verificar o processo de compartilhamento do conhecimento nas incubadoras de empresas brasileiras associadas à Associação Nacional de Entidades Promotoras de Empreendimentos de Tecnologias Avançadas (Anprotec).

Para tanto, discorre-se, inicialmente, sobre a gestão do conhecimento, o compartilhamento do conhecimento nas organizações e a conceituação de incubadoras de empresas. Em seguida, procede-se ao estudo de levantamento ou survey, analisando o compartilhamento do conhecimento nas incubadoras brasileiras associadas à Anprotec.

\section{GESTÃO DO CONHECIMENTO}

O entendimento do processo de geração do conhecimento requer, primeiramente, uma distinção entre dados, informação e conhecimento. Davenport e Prusak (I998, p. 2) definem os dados como "um conjunto de fatos distintos e objetivos, relativos a eventos. Os dados nas organizações modernas geralmente são armazenados em algum tipo de sistema tecnológico". Portanto, por ser um conjunto de fatos distintos e, muitas vezes isolados, os dados não permitem análises mais profundas acerca daquilo que se observa ou estuda. Quando relacionados ao processo decisório, os dados não fornecem uma base ideal para que se efetive a tomada de decisão.

Se, no entanto, os dados recebem algum tipo de tratamento, por meio do qual é possível que sejam realizadas determinadas inferências, têm-se as informações. Nonaka e Takeuchi (I997) ressaltam que a informação proporciona um novo ponto de vista para a interpretação de eventos, tornando visíveis significados antes invisíveis, ou lança luz sobre conexões inesperadas.

O conhecimento, por sua vez, segundo Pereira e Fonseca (I997, p. 225), "é uma forma organizada de informações consolidadas pela mente humana por meio dos mecanismos cognitivos da inteligência, da memória e da atenção". 
Apesar da distinção conceitual entre conhecimento, informação e dados, na prática são elementos interligados pelo processo de geração do conhecimento. Ainda, com relação ao conceito de conhecimento, Davenport e Prusak (i998, p. 6) comentam que:

concentra uma mistura fluida de experiência condensada, valores, informação contextual e insight experimentado, a qual proporciona uma estrutura para a avaliação e incorporação de novas experiências e informações. Ele tem origem e é aplicado na mente dos conhecedores. Nas organizações ele costuma estar embutido não só em documentos ou repositórios, mas também em rotinas, processos, práticas e normas organizacionais.

Especificamente em relação ao conceito de gestão do conhecimento, Angeloni (2002, p. XVI) explicita que "é um conjunto de processos que governa a criação, a disseminação e a utilização de conhecimento no âmbito das organizações". Complementa que a organização do conhecimento é caracterizada como aquela que acumula um repertório de saberes individuais e compartilhados pelo grupo, sendo tratado como um ativo valioso, capaz de entender e vencer as contingências ambientais.

Quando decidem trabalhar com a gestão do conhecimento, as organizações podem escolher uma ou mais opções entre as várias disponíveis. Wah (2000, p. 53) destaca alguns caminhos que podem ser utilizados para tal:

- captar, armazenar, recuperar e distribuir ativos tangíveis do conhecimento, tais como patentes ou direitos autorais;

- coletar, organizar e disseminar conhecimentos intangíveis, tais como knowhow e especialização profissional, experiência individual, soluções criativas etc.; $\mathrm{e}$

- criar um ambiente de aprendizado interativo no qual as pessoas transfiram prontamente o conhecimento, internalizem-se e apliquem-se para criar novos conhecimentos.

Nesse contexto, a gestão do conhecimento faz uso tanto do conhecimento tácito como do explícito. Segundo Nonaka e Takeuchi (I997, p. 7), o conhecimento tácito "está enraizado nas ações e experiências de um indivíduo, bem como em suas emoções, valores ou ideais. Conclusões, insights e palpites subjetivos incluem-se nesta categoria de conhecimento". Já o conhecimento explícito é aquele que pode ser verbalizado ou escrito, podendo ser transmitido facilmente entre os indivíduos. 
A gestão do conhecimento compreende, portanto, geração, armazenamento e disseminação ou compartilhamento dos vários tipos de conhecimento na organização. Considerando-se o enfoque específico deste estudo, aborda-se com mais detalhes o compartilhamento do conhecimento nas organizações.

\section{COMPARTILHAMENTO DO CONHECIMENTO NAS ORGANIZAÇÕES}

Na gestão do conhecimento, segundo Grotto (2002), um dos grandes desafios da gestão do conhecimento é promover o compartilhamento do conhecimento que não é encontrado nos manuais, nos relatórios e nas pesquisas. Por ser um conhecimento de difícil captação, formalização e comunicação, diligenciar seu compartilhamento pode requerer mudança e mobilização de toda a organização.

Saber compartilhar o conhecimento dentro da empresa, a fim de que ele não fique concentrado em algumas pessoas, apresenta-se como um desafio para os gestores. Assim, o gerenciamento eficiente do conhecimento nas organizações dependerá, primeiramente, do gerenciamento individual dos elementos que compõem a gestão do conhecimento e, posteriormente, da sinergia entre eles.

Davenport e Prusak (I998, p. XV) argumentam que "a única vantagem competitiva que uma empresa tem é aquilo que ela coletivamente sabe, a eficiência com que ela usa o que sabe e a prontidão com que ela adquire e usa novos conhecimentos". Aliado a essas questões, devem-se criar maneiras efetivas de compartilhar o conhecimento de cada indivíduo na organização.

A transferência do conhecimento envolve a transmissão e a difusão do conhecimento dentro de uma organização ou entre diferentes organizações. O compartilhamento pode ocorrer tanto entre os indivíduos que integram a organização quanto entre estes e os indivíduos externos que participam temporariamente do processo (LAHTI, 2000 apud MUSSI, 2002).

Sveiby (I998) explicita que a transferência do conhecimento pode ocorrer entre as pessoas de duas maneiras principais: informação e tradição. Pela informação, o conhecimento é transferido de forma indireta, isto é, principalmente por meio de palestras e apresentações audiovisuais. Já pela tradição, transfere-se o conhecimento de forma direta, de pessoa para pessoa, ou seja, o aprendizado pela prática.

Para Nonaka e Takeuchi (I997), o conhecimento é criado pela combinação do conhecimento tácito e do explícito, havendo modos de conversação do conhecimento: a socialização (de tácito para tácito), a externalização (de tácito para explíci- 
to), a combinação (de explícito para explícito) e a internalização (de explícito para implícito). Nessa mesma linha de raciocínio, Grotto (2002, p. I08) afirma que:

por meio dos modos de conversação do conhecimento, identificam-se os modos de compartilhamento do conhecimento tácito e explícito. Na socialização ocorre um processo de troca de experiências, pois o indivíduo compartilha seu conhecimento tácito diretamente com outro (pela linguagem, observação, imitação e prática). Na externalização, o indivíduo compartilha seu conhecimento tácito com vários outros e de forma não direta. Na combinação, um conhecimento já explícito é partilhado também de forma explícita. Na internalização, o conhecimento explícito é compartilhado de forma direta, sendo que o receptor o transforma em conhecimento implícito.

Ainda no que concerne à criação do conhecimento, Pereira (2000) apresenta um modelo de cinco fases, proposto por Nonaka e Takeuchi, envolvendo o compartilhamento do conhecimento. A primeira fase incentiva o compartilhamento do conhecimento tácito entre membros de um grupo de trabalho, que possui metas desafiadoras comuns. No processo de explicação dos conhecimentos, acontece a segunda fase, que é da criação do conhecimento, em que ocorre cristalização em conceitos explícitos decorrentes dos modelos mentais tácitos compartilhados e verbalizados. A terceira fase justifica-se pelos conceitos criados de acordo com a intenção organizacional. A quarta aproveita o conhecimento explícito recém-criado e o conhecimento explícito existente. Na quinta fase, é iniciado um novo ciclo de criação do conhecimento, que ocorre dentro da organização e entre as organizações.

Tal modelo pode servir como um guia às organizações que desejam promover ou mesmo gerenciar o processo de criação do conhecimento. Porém, quando se buscam em organizações modelos já existentes, com o intuito de servir como base, algumas ressalvas devem ser feitas, principalmente no tocante às diferenças, como: pessoas, lugares, procedimentos, ambientes, entre outros.

Davenport e Prusak (I998) entendem que algumas práticas de compartilhamento de conhecimento podem ser adotadas e, ao mesmo tempo, incentivadas nas organizações. Essas práticas objetivam um maior nível de comprometimento no compartilhamento do conhecimento. São exemplos: bebedouros e conversas; feiras e fóruns abertos do conhecimento; videoconferências; palestras, workshops e eventos; espaços sem divisórias; e outros métodos como a intranet. No que diz respeito a conversas, destaca ser esta a forma de trabalho mais importante da nova economia.

Infere-se, do exposto, a importância à atenção que deve ser dada ao compartilhamento do conhecimento em organizações que desejam efetivar a gestão do 
conhecimento. Não obstante, cabe a conscientização das pessoas sobre a necessidade de compartilhamento do conhecimento, a fim de subsidiar o crescimento de todos na organização.

Entre os vários tipos de organizações, as incubadoras já demonstram dispensar atenção especial a esse aspecto, bem como reforçam a necessidade percebida de compartilhamento do conhecimento. Na seqüência, procede-se ao entendimento do que sejam incubadoras de empresas.

\section{INCUBADORAS DE EMPRESAS}

Por causa da competitividade cada vez maior entre as empresas, alguns segmentos empresariais apresentam sinais de debilidades, como é o caso das micro e pequenas empresas. Ao contrário dessas, as grandes e médias empresas, por sua estrutura de suporte e pelo próprio poder de barganha que possuem, enfrentam melhor as características desse novo ambiente.

São, contudo, de vital importância as micro e pequenas tanto no crescimento econômico quanto na geração de novos postos de trabalho. Com base nesse contexto, o governo juntamente com entidades de vários setores, públicos ou privados, tem trabalhado na criação de mecanismos que possam apoiar esse segmento de empresas.

Dentre os mecanismos de apoio, destaca-se a atuação das incubadoras de empresas, que, além de incentivarem o desenvolvimento de negócios desse porte, buscam capacitar os empreendedores na gestão do empreendimento. Acredita-se que a empresa instalada em uma incubadora tende a apresentar maiores chances de sobrevivência, quando inserida no mercado, num comparativo com aquelas que não tiveram a mesma oportunidade. Originalmente, conforme destacam Fonseca e Kruglianskas (2000, p. 3-4):

a idéia de incubadoras esteve associada ao propósito de estimular o surgimento de negócios resultantes de projetos tecnológicos desenvolvidos no interior dos centros de pesquisa universitários ou não. O conceito criado foi o de incubadoras tecnológicas, voltadas para apoiar o nascimento e o fortalecimento das chamadas empresas de base tecnológica.

Mais recentemente, principalmente a partir dos anos I990, as iniciativas diversificaram-se. Assim, passou a ganhar espaço a idéia de incubadoras mistas, ou seja, unidades criadas por governos locais, com a finalidade de estimular o crescimento econômico e gerar empregos. 
Nesse sentido, Guedes e Formica (I997 apud AMATO NETO, 2000, p. 74) definem incubadora de empresas como:

um arranjo interinstitucional com instalações e infra-estrutura apropriadas, estruturadas para estimular e facilitar determinados fatores. São exemplos destes fatores: vinculação empresa-universidade, fortalecimento das empresas e o aumento de seu entrosamento, bem como crescente vinculação do setor produtivo com diversas instituições de apoio.

Com base nessa citação, percebe-se a crescente importância das incubadoras no ambiente empresarial, subsidiando, principalmente, o nascimento e parte do crescimento de pequenas e médias empresas. Numa analogia com o ciclo de vida das organizações, verifica-se que algumas das fases iniciais podem ser concebidas em uma incubadora.

Para a Associação Nacional de Entidades Promotoras de Empreendimentos de Tecnologias Avançadas (Anprotec, 2003):

uma incubadora de empresas é um ambiente flexível e encorajador onde é oferecida uma série de facilidades para o surgimento e crescimento de novos empreendimentos. Além da assessoria na gestão técnica e empresarial da empresa, a incubadora oferece a infra-estrutura e serviços compartilhados necessários para o desenvolvimento do novo negócio, como espaço físico, salas de reunião, telefone, fax, acesso à Internet, suporte em informática, entre outros. Desta forma, as incubadoras de empresas geridas por órgãos governamentais, universidades, associações empresariais e fundações, são catalizadoras do processo de desenvolvimento e consolidação de empreendimentos inovadores no mercado competitivo.

Infere-se do exposto que uma incubadora oferece às empresas incubadas diversas facilidades, desde apoio administrativo até auxílio na obtenção de linhas de financiamento. Mediante a infra-estrutura disponibilizada, as incubadoras de empresas contribuem para a consolidação desse setor da economia.

Conforme Baêta (I999), as incubadoras implicam condições bastante específicas, e a sua formação não se esgota numa simples criação de empresas, também há o monitoramento para estimular o processo de inovação. As incubadoras promovem oportunidades para o desenvolvimento tecnológico do processo produtivo e oferecem aos novos empreendedores, além de espaço físico e os serviços de escritório, o apoio administrativo, o aconselhamento e a consultoria gerencial e de marketing.

Sob uma perspectiva mais ampla, a missão das incubadoras é fornecer serviços e recursos compartilhados, no que se refere a profissionais competentes, 
instalações adequadas e infra-estrutura administrativa e operacional à disposição das empresas incubadas. Esses organismos criam um ambiente favorável à consolidação de novos empreendimentos por meio de algumas iniciativas, como: fornecer apoio técnico e gerencial às empresas incubadas, promover e acelerar a consolidação de empresas, estimular o espírito empreendedor, desenvolver ações associativas e compartilhadas, reduzir custos para o conjunto das empresas e seus parceiros, buscar novos apoios e parcerias para as empresas, divulgar as empresas e seus produtos e participar de outras redes (AMATO NETO, 2000).

Relacionando as várias iniciativas proporcionadas pelas incubadoras na geração de novos empreendimentos com a mortalidade precoce de muitas empresas, acredita-se que as empresas incubadas tendem a estar mais bem preparadas quando inseridas no mercado e, por conseguinte, evitam a mortalidade na fase inicial do empreendimento. Essas questões reforçam a permanência e criação de novas redes de incubadoras no País.

Quanto às tipologias de incubadoras, as mais comuns são: incubadoras de empresas de base tecnológica, incubadoras de empresas de setores tradicionais e incubadoras mistas. As incubadoras de empresas de base tecnológica contemplam empreendimentos relacionados ao desenvolvimento de tecnologia, como empresas de informática, biotecnologia, química fina, mecânica de precisão e novos materiais. As incubadoras de setores tradicionais abrigam empresas orientadas para o desenvolvimento econômico, como mecânica, eletrônica, confecção, alimentos, agroindústria, e normalmente dependem do suporte de órgãos e entidades, como prefeituras, governo do Estado ou associações comerciais, industriais e agrícolas. As incubadoras mistas, como o próprio nome demonstra, abrigam ao mesmo tempo empresas de base tecnológica e de setores tradicionais.

As incubadoras de empresas, no entanto, não atuam sozinhas. Atualmente, essas entidades desenvolvem parcerias com vários outros organismos, como governo, prefeituras, entidades com ou sem fins lucrativos, agentes financeiros, entre outros. Por meio desse apoio, as incubadoras conseguem atingir seu objetivo principal: promover o desenvolvimento e crescimento de micro e pequenas empresas.

Aliado à definição de incubadoras, tem-se discutido o compartilhamento do conhecimento no ambiente no qual as empresas encontram-se incubadas. Devese analisar não somente o compartilhamento do conhecimento entre os gestores e empregados da incubadora, entre os empregados e os empreendedores, mas, principalmente, a troca de conhecimentos entre empreendedores de ramos similares ou de ramos diferentes, que muitas vezes detectam a mesma dificuldade durante o processo de incubação.

Por ser um ambiente em que são gerados conhecimentos, os gestores das incubadoras de empresas precisam canalizá-los, por intermédio dos empreen- 
dedores, para que possam ser difundidos e desenvolvidos por meio de novas unidades de negócio. Assim, diante do exposto, optou-se por analisar o processo de compartilhamento do conhecimento nas incubadoras brasileiras associadas à Anprotec.

\section{METODOLOGIA DA PESQUISA}

A preocupação com o conhecimento da realidade é uma constante na vida do homem. A pesquisa apresenta-se como uma forma de investigação que tem como finalidade buscar respostas às indagações da sociedade, por meio de procedimentos científicos. Os seus delineamentos possuem um importante papel na pesquisa científica para articular planos e estruturas, a fim de obter respostas para os problemas de estudo.

Os delineamentos desta pesquisa deram-se em razão dos objetivos, dos procedimentos e da abordagem do problema. No que diz respeito aos objetivos, esta pesquisa consiste em um estudo do tipo descritivo. No que concerne aos procedimentos, refere-se a uma pesquisa do tipo levantamento ou survey. Quanto à abordagem do problema, o estudo utilizou-se da abordagem quanti-qualitativa.

A população compõe-se de I86 incubadoras de empresas brasileiras extraídas da relação disponibilizada pela equipe de pesquisa da Associação Nacional de Entidades Promotoras de Empreendimentos de Tecnologias Avançadas (Anprotec), no site da referida associação (http://www.anprotec.org.br).

Pela impossibilidade de analisar todas as incubadoras, optou-se por selecionar uma amostra. A restrição deve-se em virtude de vários fatores, como: falta de disponibilidade de tempo e interesse de todas as incubadoras e tempo limitado para a conclusão da pesquisa. Assim, adotou-se o conceito de amostragem, considerando como amostra o retorno dos questionários respondidos de 33 incubadoras brasileiras.

Para a coleta de dados, foi utilizada a técnica de questionários, com perguntas abertas e fechadas. Gil (I999) explicita que o questionário, como instrumento de coleta de dados, é composto de um número mais ou menos elevado de questões apresentadas por escrito às pessoas, tendo como objetivo o conhecimento de suas opiniões, crenças, sentimentos, interesses, expectativas, situações vivenciadas.

Os questionários foram enviados por correio eletrônico, no mês de novembro de 2004, aos coordenadores das incubadoras. Dos I86 questionários enviados, obteve-se um retorno de 33 incubadoras, os quais representam a amostra por acessibilidade extraída da população. 
No tocante à documentação indireta, como fonte de coleta de dados, utilizouse a técnica da pesquisa bibliográfica e da pesquisa documental. Com relação à última, foi utilizada a investigação de informações disponibilizadas nos sites das incubadoras que participaram do estudo.

A análise dos dados coletados da pesquisa deu-se por meio da abordagem quanti-qualitativa. Para tanto, foram utilizadas as técnicas de análise descritiva e documental. As técnicas de análise dos dados abordadas são amplamente empregadas nas Ciências Sociais, em virtude de diversos trabalhos nessa área demandarem uma abordagem quanti-qualitativa.

\section{DESCRIÇÃO E ANÁLISE dOS DADOS COLETADOS}

A verificação do processo de compartilhamento do conhecimento nas incubadoras de empresas brasileiras associadas à Anprotec deu-se pela tabulação das respostas dos questionários enviados via correio eletrônico aos coordenadores das incubadoras.

Antes de proceder à análise do compartilhamento do conhecimento, faz-se necessário evidenciar a compreensão dos gestores das incubadoras em relação à gestão do conhecimento. Na Tabela I, apresentam-se características acerca da compreensão dos coordenadores das incubadoras sobre a gestão do conhecimento.

Pode-se inferir que os gestores das incubadoras possuem bom entendimento sobre a gestão do conhecimento. Entre os sujeitos da pesquisa, $70 \%$ responderam ter bom entendimento.

A segunda pergunta referiu-se ao interesse dos entrevistados em aprender ou conhecer mais sobre a gestão do conhecimento. Percebeu-se que, apesar do bom entendimento apontado, os gestores, na sua quase totalidade, têm grande interesse em aprender ou conhecer mais sobre a gestão do conhecimento, o que mostra a sua consciência sobre o papel das incubadoras junto aos incubados.

A terceira questão relacionou-se ao uso de formas de interação entre os empregados da incubadora. A existência ou não de formas de interação vai interferir no grau de compartilhamento do conhecimento na organização. Assim, quando questionados se a incubadora usa formas de interação entre seus empregados, a maioria dos coordenadores indicou a alternativa bom.

Com vistas no foco mais específico da pesquisa, procedeu-se à identificação do grau de importância para cada forma de compartilhamento do conhecimento pelos coordenadores. Portanto, na quarta questão procurou-se identificar o grau de importância, para os respondentes, das formas de compartilhamento do co- 
nhecimento, sendo o o mínimo e 4 o máximo. Foram relacionadas as formas mais comuns de compartilhamento do conhecimento no âmbito das organizações, contempladas na literatura. Esses itens, com suas respectivas notas, são apresentados na Tabela 2.

\section{TABELA I}

COMPREENSÃO DOS COORDENADORES DAS INCUBADORAS SOBRE A GESTÃO DO CONHECIMENTO

\begin{tabular}{lcc}
\hline QUESTÕES & RESPOSTAS & PORCENTAGEM \\
\hline $\begin{array}{l}\text { 1. Como classificaria seu entendimento sobre a } \\
\text { gestão do conhecimento? }\end{array}$ & Nenhum & $0 \%$ \\
& Pouco & $18 \%$ \\
& Bom & $70 \%$ \\
\hline $\begin{array}{l}\text { Total da questão } \\
\text { Qu conhecer mais sobre a gestão do }\end{array}$ & Muito bom & $12 \%$ \\
\hline conhecimento? & Grande & $100 \%$ \\
\hline $\begin{array}{l}\text { 2. } \\
\text { Total da questão }\end{array}$ & Médio & $91 \%$ \\
\hline seus empregados? & Pequeno & $9 \%$ \\
\hline Incubadora usa formas de interação entre & Indiferente & $0 \%$ \\
\hline & & $10 \%$ \\
\hline
\end{tabular}

Fonte: Dados da pesquisa. 


\section{TABELA 2}

GRAU DE IMPORTANCIA PARA CADA FORMA DE COMPARTILHAMENTO DO CONHECIMENTO

\begin{tabular}{lc} 
FORMAS & MÉDIA DAS NOTAS \\
\hline 1. Reunião & 3,3 \\
\hline 2. Encontros & 3,3 \\
\hline 3. Palestras & 3,4 \\
\hline 4. Intranet & 2,9 \\
\hline 5. Seminários & 3,1 \\
\hline 6. Discussões & 3,3 \\
\hline 7. Livros & 2,8 \\
\hline 8. Revistas & 2,7 \\
\hline 9. Jornais & 2,8 \\
\hline 10. Realização de pesquisas & 3,2 \\
\hline
\end{tabular}

Fonte: Dados da pesquisa.

Das formas analisadas, as que apresentam maior grau de importância, na média, são as palestras, as reuniões, os encontros e discussões. Infere-se que a intranet, apesar de ser um meio de comunicação bastante utilizado entre os empregados e gestores, na maioria das organizações, não ocupou destaque no ranking, ficando na quinta posição.

Pode-se observar, no entanto, que nenhuma das formas de compartilhamento do conhecimento ficou com uma nota média abaixo de 2 . Ressalta-se ainda que, mesmo que não sejam utilizadas com muita intensidade pelas incubadoras, as demais formas apresentam relevância de acordo com a opinião dos entrevistados.

Depois de identificado o grau de importância atribuído às formas de compartilhamento do conhecimento, questionou-se sobre a eficiência, freqüência e participação em discussões de assuntos profissionais da incubadora. As discussões entre os empregados e gestores promovidas no âmbito das organizações representam, além de uma forma importante de disseminação, uma forma de 
geração do conhecimento. Na Tabela 3, evidenciam-se a eficiência, freqüência e participação dos empregados da incubadora nas discussões, conforme as respostas dos coordenadores.

\section{TABELA 3}

EFICIENCIA, FREQUUENCIA E PARTICIPAÇÂO DOS EMPREGADOS NAS DISCUSSÕES

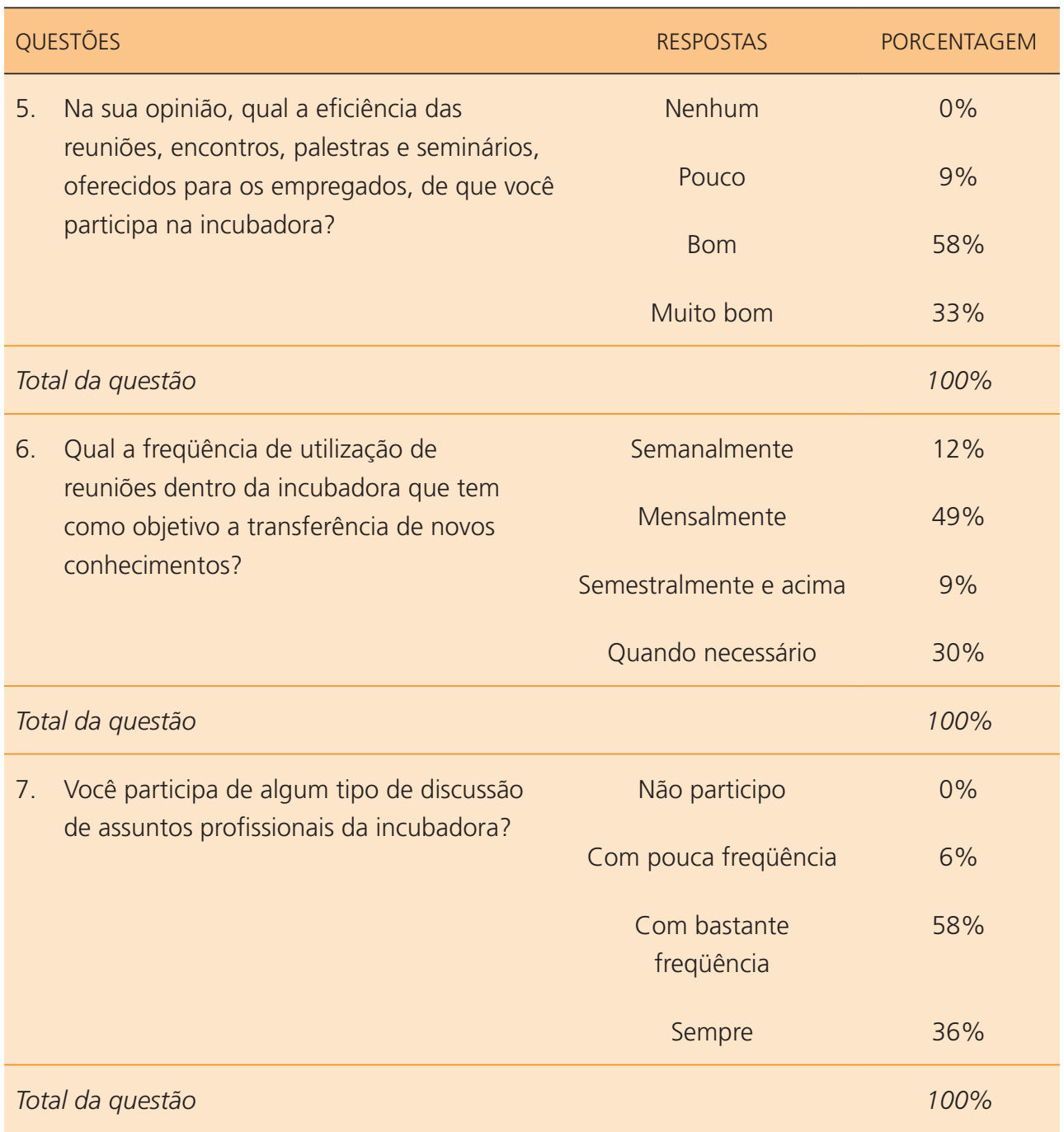

Fonte: Dados da pesquisa. 
A quinta questão solicitou a opinião dos coordenadores das incubadoras sobre a eficiência das reuniões, palestras e dos seminários oferecidos aos seus empregados. Eles apontaram que a utilização de reuniões, palestras e seminários traz uma eficiência boa, com $58 \%$ das indicações. Isso demonstra a importância de efetivar essas atividades dentro das organizações, pois elas permitem que os empregados vivenciem novas experiências, o que promove a troca de conhecimento.

A questão seguinte indagou sobre a freqüência de reuniões dentro da incubadora, evento que tem como objetivo a transferência de novos conhecimentos. Os resultados mostram que as reuniões ocorrem mensalmente para a interação dos empregados da incubadora, com $49 \%$ dos casos. Todavia, $30 \%$ dos sujeitos da pesquisa responderam que essas reuniões são realizadas apenas quando necessário.

A sétima questão investigou a participação do respondente em discussões sobre assuntos profissionais da incubadora. A opção com bastante freqüência representa $58 \%$ das respostas. Apenas $6 \%$ dos respondentes indicaram que participam com pouca freqüência dessas discussões.

Também se procurou verificar quais são os lugares que mais contribuem para essas discussões ocorrerem, atribuindo uma nota de o a 4, sendo o o mínimo e 4 o máximo. Os lugares elencados foram: durante o serviço; no cafezinho, almoço e lanche; após o trabalho; e em outros encontros, como futebol e festas. Apresentam-se na Tabela 4 as notas atribuídas pelos entrevistados para cada opção e as médias obtidas.

\section{TABELA 4}

\section{LUGARES QUE MAIS CONTRIBUEM PARA AS DISCUSSÖES}

\section{LUGARES}

1. Durante o serviço

2. No cafezinho, almoço, lanche

3. Após o trabalho

4. Em outros encontros, como futebol e festas
MÉDIA DAS NOTAS

\section{3,6}

2,5

1,6

1,1

Fonte: Dados da pesquisa.

Observa-se que o local mais apropriado para que ocorram as discussões é o próprio ambiente de trabalho. Contudo, apesar de os demais lugares não serem 
os que mais contribuem no caso das incubadoras, muitas vezes é nesses lugares que ocorre a troca de idéias e é nesses espaços que elas são desenvolvidas.

Características do compartilhamento do conhecimento também foram pesquisadas nas incubadoras de empresas que participaram do estudo. Na Tabela 5, apresenta-se a percepção dos coordenadores das incubadoras pesquisadas quanto a essas características.

$\mathrm{Na}$ nona questão perguntou-se qual o efeito positivo dos debates promovidos no ambiente da incubadora, quando estes existem. Quando ocorre interação entre as pessoas, de alguma maneira isso vai contribuir para a organização, pois a troca de informações irá estimular o conhecimento de cada um. Dos sujeitos da pesquisa, $67 \%$ responderam que o efeito positivo é bom e 30\% responderam muito bom.

A pergunta seguinte buscou verificar se os coordenadores das incubadoras de empresas compartilham seus conhecimentos com outras pessoas. Por serem os coordenadores das incubadoras de empresas, devem ser os primeiros a incentivar essa forma de transferência do conhecimento. Observou-se que 9i\% dos respondentes informaram que sempre compartilham seus conhecimentos com outras pessoas da incubadora, ajudando na disseminação do conhecimento. Apenas $3 \%$ responderam que isso ocorre só quando perguntam.

Na seqüência, a pergunta onze questionou se a incubadora busca pessoas especializadas para a realização de palestras ou seminários. A busca de novas experiências, com pessoas vindas de outros lugares, é muito importante para a geração e troca de conhecimento. Verifica-se que $46 \%$ dos respondentes disseram utilizar essa forma com bastante freqüência, 45\% com razoável freqüência e 9\% com pouca freqüência.

A questão doze buscou saber se existe a participação de empregados em encontros regionais e nacionais na busca de novos conhecimentos. Como as organizações estão sendo impulsionadas a contar cada vez mais com pessoas especializadas para manterem-se competitivas, torna-se relevante a participação dos empregados e gestores em encontros que abordam assuntos de interesse destes e/ou da incubadora. Dos respondentes, 34\% informaram que isso ocorre com bastante freqüência, 45\% com razoável freqüência e 21\% com pouca freqüência.

A última pergunta do questionário está atrelada às respostas da questão anterior. Perguntou-se de que maneira a incubadora proporciona o compartilhamento do conhecimento adquirido pelas pessoas que participaram desses encontros. Nota-se que 55\% responderam que as pessoas que participam de encontros regionais ou nacionais compartilham o conhecimento através de reuniões, e somente $2 \%$ não compartilham. 


\section{TABELA 5}

CARACTERIISTICAS DO COMPARTILHAMENTO DO CONHECIMENTO NAS INCUBADORAS

\begin{tabular}{|c|c|c|}
\hline QUESTÕES & RESPOSTAS & PORCENTAGEM \\
\hline \multirow{4}{*}{$\begin{array}{l}\text { 9. Qual é o efeito positivo dos debates na } \\
\text { incubadora, quando existem? }\end{array}$} & Nenhum & $0 \%$ \\
\hline & Pouco & $3 \%$ \\
\hline & Bom & $67 \%$ \\
\hline & Muito bom & $30 \%$ \\
\hline \multicolumn{2}{|l|}{ Total da questão } & $100 \%$ \\
\hline \multirow{4}{*}{$\begin{array}{l}\text { 10. Você compartilha seus conhecimentos com } \\
\text { outras pessoas da incubadora? }\end{array}$} & Sempre & $91 \%$ \\
\hline & Com pouca freqüência & $6 \%$ \\
\hline & Só quando perguntam & $3 \%$ \\
\hline & Não & $0 \%$ \\
\hline \multicolumn{2}{|l|}{ Total da questão } & $100 \%$ \\
\hline \multirow{4}{*}{$\begin{array}{l}\text { 11. A incubadora busca pessoas especializadas } \\
\text { para a realização de palestras ou } \\
\text { seminários? }\end{array}$} & $\begin{array}{l}\text { Sim, com bastante } \\
\text { freqüência }\end{array}$ & $46 \%$ \\
\hline & $\begin{array}{l}\text { Sim, com razoável } \\
\text { freqüência }\end{array}$ & $45 \%$ \\
\hline & $\begin{array}{l}\text { Sim, com pouca } \\
\text { freqüência }\end{array}$ & $9 \%$ \\
\hline & Não acha necessário & $0 \%$ \\
\hline \multicolumn{2}{|l|}{ Total da questão } & $100 \%$ \\
\hline \multirow{4}{*}{$\begin{array}{l}\text { 12. Existe a participação de empregados em } \\
\text { encontros regionais e nacionais na busca } \\
\text { de novos conhecimentos? }\end{array}$} & $\begin{array}{l}\text { Sim, com bastante } \\
\text { freqüência }\end{array}$ & $34 \%$ \\
\hline & $\begin{array}{l}\text { Sim, com razoável } \\
\text { freqüência }\end{array}$ & $45 \%$ \\
\hline & $\begin{array}{l}\text { Sim, com pouca } \\
\text { freqüência }\end{array}$ & $21 \%$ \\
\hline & Não acha necessário & $0 \%$ \\
\hline \multicolumn{2}{|l|}{ Total da questão } & $100 \%$ \\
\hline \multirow{4}{*}{$\begin{array}{l}\text { 13. Sendo a resposta acima afirmativa, de } \\
\text { que maneira a incubadora proporciona } \\
\text { o compartilhamento do conhecimento } \\
\text { dessas pessoas? }\end{array}$} & Através de documentos & $12 \%$ \\
\hline & Através de reuniões & $55 \%$ \\
\hline & Promovendo palestras & $31 \%$ \\
\hline & Não compartilha & $2 \%$ \\
\hline \multicolumn{2}{|l|}{ Total da questão } & $100 \%$ \\
\hline
\end{tabular}

Fonte: Dados da pesquisa. 
Nesse sentido, verificou-se o processo de compartilhamento do conhecimento nas incubadoras de empresas pesquisadas. Observou-se que, visando o alcance de seus objetivos organizacionais, as incubadoras buscam na gestão do conhecimento, particularmente no compartilhamento do conhecimento, uma maneira de efetivar a geração e a disseminação do conhecimento. Assim, podem preparar melhor seus empregados, desenvolver as empresas incubadas, bem como potencializar as características dos empreendedores nelas instalados.

\section{CONCLUSÕES}

O ambiente competitivo em que as empresas encontram-se inseridas está continuamente em modificação. Nesse contexto, é de fundamental importância a preocupação com a gestão do conhecimento, para organizar os processos que administram a criação, disseminação e utilização do conhecimento, a fim de atingir plenamente os objetivos organizacionais. Com base na gestão do conhecimento, é possível melhorar a maximização e disponibilização dos conhecimentos existentes na empresa, além de fazer que ele esteja à disposição de todos na organização.

Entre os aspectos relacionados à gestão do conhecimento, este estudo deu ênfase ao compartilhamento do conhecimento. A predisposição pelo compartilhamento dos conhecimentos individuais é essencial para que haja a formação do conhecimento na organização, haja vista que quem os detém decide se os compartilha ou não.

Nesse sentido, este artigo buscou relatar os resultados de um estudo realizado nas incubadoras brasileiras associadas à Associação Nacional de Entidades Promotoras de Tecnologias Avançadas (Anprotec), com a finalidade de analisar o processo de compartilhamento do conhecimento.

Nas incubadoras estudadas, observou-se que há uma preocupação em gerar, difundir e, sobretudo, compartilhar informações e conhecimentos. Isso para que as empresas incubadas tenham condições, em um período relativamente curto de tempo, de se tornarem empresas capazes de competir com outras do mesmo ramo, setor ou atividade, independentemente de sua região ou país de origem.

Quanto às formas de compartilhamento analisadas, verificou-se que as que apresentam maior grau de importância nas incubadoras de empresas são as palestras, as reuniões, os encontros e as discussões. Notou-se que a intranet, apesar da facilidade de acesso como meio de comunicação, ocupou o quinto lugar no ranking das modalidades apresentadas. Contudo, nenhuma das formas de compartilhamento do conhecimento ficou com nota média abaixo de 2, numa 
escala de $\circ$ a 4. Mesmo que não sejam utilizadas com muita intensidade pelas incubadoras, as demais formas apresentam relevância de acordo com a opinião dos respondentes.

No que concerne à eficiência, freqüência e participação em discussões, constatou-se que, na percepção dos coordenadores, é de suma importância a realização dessas atividades dentro das organizações, trazendo novas experiências, bem como troca de conhecimento. Além disso, percebeu-se que as reuniões são consideradas de grande importância para a interação entre os empregados das incubadoras.

Em relação aos lugares que mais contribuem para as discussões, segundo os respondentes do questionário, o local mais apropriado para que isso ocorra é o próprio ambiente de trabalho. Todavia, apesar de os demais lugares não serem os que mais contribuem para as incubadoras pesquisadas, é nesses lugares que, muitas vezes, ocorrem as discussões.

No tocante aos debates promovidos, observou-se que estes, quando existem, geram um efeito positivo no ambiente da incubadora. Foi destacado que os empregados normalmente compartilham os conhecimentos adquiridos, ajudando na disseminação do conhecimento.

Por fim, verificou-se que as incubadoras buscam pessoas especializadas para a realização de palestras ou seminários. Percebeu-se que a busca de novas experiências com pessoas vindas de outros lugares foi considerada importante pelos respondentes para a geração e troca de conhecimento. Além disso, as incubadoras incentivam a participação dos empregados em encontros regionais e nacionais, na busca de novos conhecimentos.

Assim, conclui-se que os resultados obtidos junto ao estudo realizado nas incubadoras brasileiras associadas à Anprotec apresentam as características de compartilhamento do conhecimento nessas organizações.

Os conceitos preconizados pela teoria acerca da gestão do conhecimento e, particularmente, em relação ao compartilhamento do conhecimento foram identificados na pesquisa realizada. Os gestores e empregados das incubadoras de empresas já percebem a importância que deve ser dada ao compartilhamento do conhecimento, a fim de efetivar a gestão do conhecimento. Não obstante, buscam a conscientização das pessoas sobre a necessidade de compartilhamento do conhecimento, a fim de subsidiar o crescimento de todos na organização. 


\section{REFERÊNCIAS}

AMATO NETO, João. Redes de cooperação produtiva e clusters regionais: oportunidades para as pequenas e médias empresas. São Paulo: Atlas, Fundação Vanzolini, 2000.

ANGELONI, Maria Terezinha (Coord.). Organizações do conhecimento: infra-estrutura, pessoas e tecnologias. São Paulo: Saraiva, 2002.

ASSOCIAÇÃO NACIONAL DE ENTIDADES PROMOTORAS DE EMPREENDIMENTOS DE TECNOLOGIAS AVANÇADAS - Anprotec. Incubadora de empresas. Disponível em: <http:// www.anprotec.org.br/anprotec.htm\#5>. Acesso em: 9 dez. 2003.

BAÊTA, Adelaide Maria Coelho. O desafio da criação: uma análise das incubadoras de empresas de base tecnológica. Petrópolis: Vozes, I999.

DAVENPORT, Thomas; PRUSAK, Laurence. Conhecimento empresarial: como as organizações gerenciam o seu capital intelectual. Rio de Janeiro: Campus, I998.

FONSECA, Sérgio Azevedo; KRUGLIANSKAS, Isak. Avaliação do desempenho de incubadoras empresariais mistas: um estudo de caso no Estado de São Paulo, Brasil. In: IASP - CONFERÊNCIA LATINO-AMERICANA DE PARQUES TECNOLÓGICOS E INCUBADORAS DE EMPRESAS, 2000, Panamá. Anais... Panamá: Iasp, 2000. CD-ROM.

GIL, Antônio Carlos. Métodos e técnicas de pesquisa social. 5. ed. São Paulo: Atlas, I999.

GROTTO, Daniela. O compartilhamento do conhecimento nas organizações. In: ANGELONI, Maria Terezinha (Coord.). Organizações do conhecimento: infra-estrutura, pessoas e tecnologias. São Paulo: Saraiva, 2002.

MUSSI, Clarissa Carneiro. O compartilhamento do conhecimento no processo de implementação de sistemas integrados de informação: o caso da Universidade do Sul de Santa Catarina. 2000. I85 f. Dissertação (Mestrado em Administração) - Programa de Pós-Graduação em Administração, Universidade Federal de Santa Catarina, Florianópolis, 2000.

NONAKA, Ikujiro Oliveira; TAKEUCHI, Hitotaka. Criação de conhecimento na empresa: como as empresas geram a dinâmica da organização. 2. ed. Rio de Janeiro: Campus, I997.

PEREIRA, Ricardo Oliveira. Gestão do conhecimento na indústria: uma proposta de avaliação da gestão do conhecimento para a indústria do setor moveleiro da região metropolitana de Curitiba. 2000. 99 f. Dissertação (Mestrado em Engenharia de Produção) - Programa de Pós-Graduação em Engenharia de Produção, Universidade Federal de Santa Catarina, Florianópolis, 2000.

PEREIRA, M. J. B. P.; FONSECA, J. G. M. Faces da decisão: as mudanças de paradigmas e o poder da decisão. São Paulo: Makron Books, I997.

SVEIBY, Karl Erik. A nova riqueza das organizações. Rio de Janeiro: Campus, I998.

WAH, Louisa. Muito além de um modismo. HSM Management, São Paulo, ano 4, n. 22, p. 52-64, set./out. 2000.

\section{TRAMITAÇÃO}

Recebido em 11/4/2005

Aprovado em 16/10/2006 


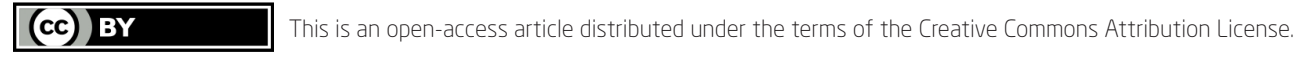

This paper may be copied, distributed, displayed, transmitted or adapted if provided, in a clear and explicit way, the name of the journal, the edition, the year and the pages on which the paper was originally published, but not suggesting that RAM endorses paper reuse. This licensing term should be made explicit in cases of reuse or distribution to third parties. It is not allowed the use for commercial purposes.

Este artigo pode ser copiado, distribuído, exibido, transmitido ou adaptado desde que citados, de forma clara e explícita, o nome da revista, a edição, o ano e as páginas nas quais o artigo foi publicado originalmente, mas sem sugerir que a RAM endosse a reutilização do artigo. Esse termo de licenciamento deve ser explicitado para os casos de reutilização ou distribuição para terceiros. Não é permitido o uso para fins comerciais. 